\title{
Sparse Low-Rank Based Signal Analysis Method for Bearing Fault Feature Extraction
}

\author{
Baoxiang Wang ${ }^{1,2}{ }^{-}$, Yuhe Liao ${ }^{1,2, *}$, Rongkai Duan ${ }^{1,2}$ and Xining Zhang ${ }^{2}$ \\ 1 Key Laboratory of Education Ministry for Modern Design and Rotor-Bearing System, Xi'an Jiaotong \\ University, Xi'an 710049, China; wangbaoxiang@stu.xjtu.edu.cn (B.W.); duanrongkai@stu.xjtu.edu.cn (R.D.) \\ 2 Shaanxi Key Laboratory of Mechanical Product Quality Assurance and Diagnostics, Xi'an Jiaotong \\ University, Xi'an, Shaanxi 710049, China; Zhang-xining@163.com \\ * Correspondence: yhliao@xitu.edu.cn
}

Received: 9 February 2020; Accepted: 25 March 2020; Published: 30 March 2020

Featured Application: This work is applied to condition detection and fault diagnosis of rotating machinery.

\begin{abstract}
The condition monitoring of rolling element bearings (REBs) is essential to maintain the reliable operation of rotating machinery, and the difficulty lies in how to estimate fault information from the raw signal that is always overwhelmed by severe background noise and other interferences. The method based on a sparse model has attracted increasing attention because it can capture deep-level fault features. However, when processing a signal with complex components and weak fault features, the performance of sparse model-based methods is often not ideal. In this work, the fault information-based sparse low-rank algorithm (FISLRA) is proposed to abstract the fault information from a noisy signal interfered with by background noise and external interference. Concretely, a sparse and low-rank model is formulated in the time-frequency domain. Then, a fast-converging algorithm is derived based on the alternating direction method of multipliers (ADMM) to solve the formulated model. Moreover, to further highlight the periodical transients, a correlated kurtosis-based thresholding (CKT) scheme proposed in this paper is also incorporated to solve the proposed low-rank spares model. The superiority of the proposed FISLRA over the traditional sparse low-rank model (TSLRM) and spectral kurtosis (SK) is proved by simulation analysis. In addition, two experimental signals collected from a bearing test rig are utilized to demonstrate the efficiency of the proposed FISLRA in fault detection. The results illustrate that compared to the TSLRM method, FISLRA can effectively extract periodical fault transients even when harmonic components (HCs) are present in the noisy signal.
\end{abstract}

Keywords: fault features extraction; bearing fault diagnosis; correlated kurtosis-based thresholding (CKT) scheme; fault information based sparse low-rank algorithm (FISLRA)

\section{Introduction}

To avoid the occurrence of unexpected shutdowns, condition assessment and fault detection methods are widely used in industrial applications [1-5]. The condition monitoring and fault diagnosis of important mechanical components are the basis of the condition assessment of mechanical equipment. A rolling bearing is an indispensable supporting part of various machines, such as wind turbines [6], automobiles, helicopters [7], driving motors [8], etc. Due to the tough operating conditions, rolling element bearings (REBs) inevitably suffer from unexpected failures and these failures may result in machine breakdown and economic losses $[9,10]$. Therefore, the condition monitoring of REBs has attracted considerable attention from academia and industry [11,12]. 
Vibration analysis is known as an effective means of structural damage detection, and thus has become one of the most extensively applied methods. When a defect occurs on the surface of REBs, repetitive transients with a particular period will be generated [13]. The occurrence of repetitive transients is significant evidence for bearing failure. Therefore, the extraction of repetitive transients is essential for bearing fault diagnosis. However, due to the harsh operating environment, the repetitive transients are usually submerged by environmental noise, specifically when the bearing fault is at an early stage. An increasingly complex structure of rotating machinery and a harsh operating environment also make the extraction of fault features more difficult.

Many scholars have spared no effort to extract the weak repetitive transients, and some fruitful diagnostic methods are proposed such as, wavelet transform (WT) [13,14], spectral kurtosis (SK) [15,16], variational mode decomposition (VMD) [17], empirical mode decomposition (EMD) [18,19], time-frequency analysis [20], sparse representation (SR) [21-25] and so on. Practice has proved that these methods have been successfully applied to condition monitoring of REBs in industrial fields.

By contrast with traditional bearing fault diagnosis methods, a sparsity-based algorithm can recover higher level fault information from a noisy signal, thus it has received considerable attention for its strong performance for detecting the rolling bearing fault in recent years. For instance, Chen and Selesnick [26,27] proposed overlapping group shrinkage (OGS)-based optimization models to estimate the group-sparse signal from a noisy signal. Furthermore, He [22] and Ding [28] proposed improved OGS-based algorithms for the extraction of oscillatory features generated by rotating machinery. The tunable-Q wavelet transform (TQWT)-based method raised by Cai [29] and Wang [30] has been applied for the extraction of fault information of a gearbox and bearing, respectively. In addition, to further facilitate the sparsity of the solution, non-convex penalty functions (NPFs) are introduced into the sparse model $[28,31,32]$. The above analysis shows that a sparse-assisted algorithm is successfully applied in bearing structural damage detection. However, the sparse model-based algorithm needs to be further studied in processing signals with complex components and weak fault features.

The sparse low-rank model-based method has made remarkable achievements in many fields, such as graph denoising [33], subspace clustering [34], image classification [35] and so on. In addition, the sparse low-rank model-based algorithm can extract fault features more effectively since it can take into account the low rank characteristic and sparsity of the bearing fault features simultaneously [36]. Therefore, the sparse low-rank model-based signal processing method has shown great potential for the fault detection of REBs. However, so far the research on applying the sparse low-rank model to bearing defect detection is in the preliminary stage. It is found that the extraction of fault features via directly applying the traditional sparse low-rank model without considering the characteristics of bearing vibration signal is susceptible to interference from harmonic components (HCs).

In this work, to effectively extract the periodical fault transients even when HCs present in the noisy signal, a fault information-based sparse low-rank algorithm (FISLRA) is proposed. Concretely, a sparse and low-rank model is formulated in the time-frequency domain. Then, a fast converging algorithm is derived based on the alternating direction method of multipliers (ADMM) to solve the formulated model. Moreover, to further highlight the periodical transients, the correlated kurtosis-based thresholding (CKT) scheme proposed in this paper is also incorporated to solve the proposed low-rank spare model, and a parameter selection scheme is presented in detail. The performance of FISLRA is validated by simulated and real vibration signals while being compared with other state-of-the-art methods. The results indicate that FISLRA can accurately extract the fault transients from noisy signals and achieve better performance than other comparison methods.

The main contributions of the paper are described as follows:

1. A fault information-based sparse low-rank algorithm (FISLRA) is proposed and applied for fault feature extraction of rolling bearing.

2. A correlated kurtosis-based thresholding (CKT) scheme is proposed and it is incorporated to solve the proposed low-rank spare model. 
The remaining parts of this paper are organized as follows: Section 2 introduces the prior knowledge of sparsity and low-rank characteristic. Section 3 introduces the proposed FISLRA. In Section 4, the simulation analysis is implemented for the verification of the proposed FISLRA. In Section 5, experimental analysis is performed. The conclusion is summarized in Section 6.

\section{Prior Knowledge of Sparsity and Low-Rank Characteristic}

In stationary operating conditions, periodic impulses as displayed in Figure 1 will be produced if a defect occurs on the bearing outer. The repetitive impulses show sparsity in the time domain. Moreover, the repetitive impulses excite resonance of the bearing, and there are similarities among the resonances. Meanwhile, the time-frequency diagram of the repetitive impulses after short-time Fourier transform (STFT) is shown in Figure 2. The similarity among the STFT coefficients of the repetitive impulses in the resonance band makes the STFT coefficient matrix show a low rank characteristic. The coefficients are sparse in the time-frequency diagram. However, the environmental noise does not show a low-rank characteristic and sparsity. Therefore, a low-rank sparse model based on prior knowledge of sparsity and a low rank characteristic has great potential for fault feature extraction from a noisy signal.

(a)

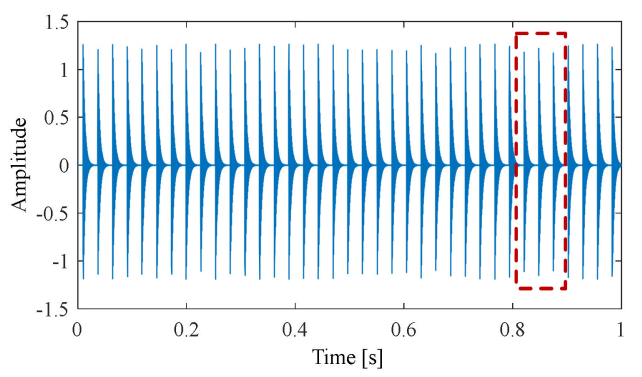

(b)

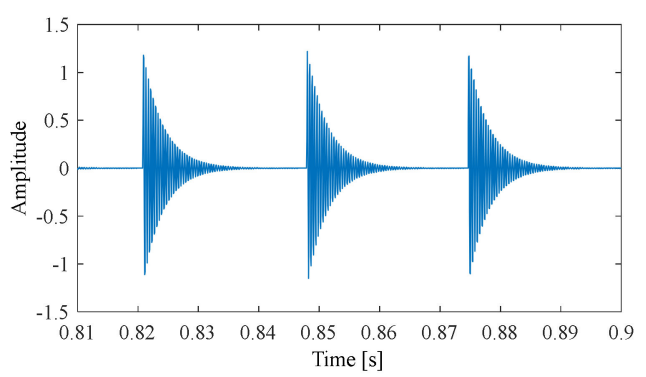

Figure 1. (a) Periodic impulsive features; (b) a partially enlarged view of (a).

(a)

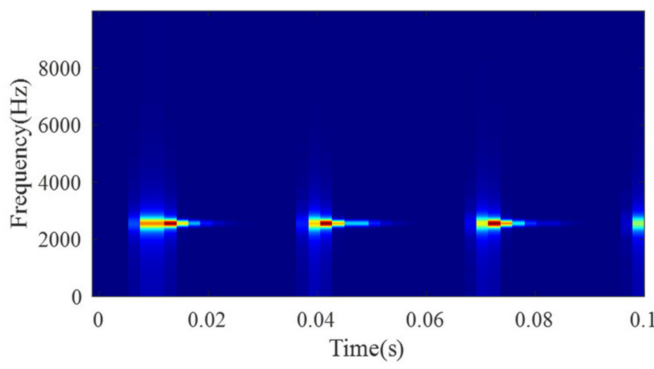

(b)

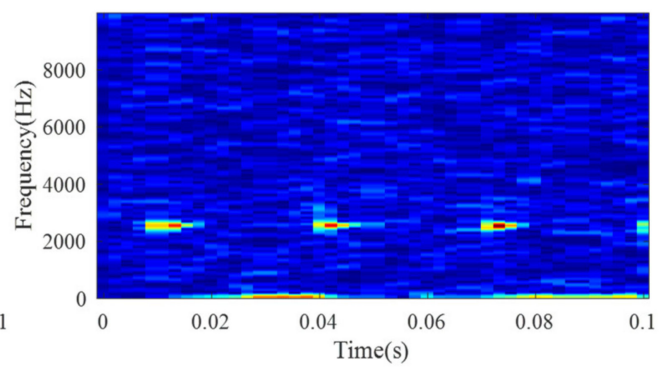

Figure 2. Time-frequency diagram: (a) periodic impulsive features; (b) noisy signal.

\section{The Proposed Fault Information-Based Sparse Low-Rank Algorithm}

The sparsity and low-rank characteristics of the fault features are explored in Section 2. To abstract the STFT coefficient matrix $X$ corresponding to the fault information from the noisy matrix $Y$, the TSLRM can be defined as:

$$
\arg \min _{X \in R^{m \times n}}\left\{\|Y-X\|_{F}^{2}+\lambda_{0}\|X\|_{*}+\lambda_{1}\|X\|_{1}\right\}
$$

where $\|X\|_{*}$ and $\|X\|_{1}$ are the trace norm and $l_{1}$ norm, respectively. $\lambda_{i} \geq 0$ represents the regularization parameter. In Equation (1) trace norm $\|X\|_{*}$ and $\|X\|_{1}$ promote sparsity of singular values (SVs) and elements of matrix $X$, respectively. Essentially, the trace norm is the $l_{1}$ norm of the SVs of the matrix $X$. The signal analysis method based on the TSLRM has been widely applied in graph denoising. However, TSLRM is insufficient in the bearing fault diagnosis field, since the bearing fault information is usually submerged by external interference. In particular, TSLRM is susceptible to the interference 
from the HCs generated by the parts such as gears or rotors. To overcome this difficulty and to improve the capability of fault feature extraction, a FISLRA is proposed. In the FISLRA, the corresponding sparse and low-rank model can be designed as follows:

$$
X^{*}=\arg \min _{X \in R^{m \times n}}\left\{F(X):=\frac{1}{2}\|Y-X\|_{F}^{2}+\lambda_{0} \sum_{i=1}^{k} \sigma_{i}(X)+\lambda_{1} \sum_{m_{1}=1}^{M_{1}} \sum_{m_{2}=1}^{M_{2}} \phi\left(X_{m_{1}, m_{2}} ; a\right)\right\}
$$

where Xand $Y \in C^{M_{1} \times M_{2}}$ are the STFT coefficient matrix; $k=\min \left(M_{1}, M_{2}\right)$. Note that the FISLRA is a signal processing technique based on matrix estimation, so the raw signal needs to be converted into a matrix $Y$ through the STFT operator $A^{T}$. The transformation relation is indicated as $Y=A^{T} y$. The parameter settings of STFT in this paper are as follows: window size $\mathrm{R}=64$ with $50 \%$ window overlapping, and the length of Fourier transform is $\mathrm{L}=256$. Moreover, the STFT coefficient matrix $X^{*}$ is the solution of optimization problem (2). Finally, obtaining the estimated fault feature $x$ through inverse STFT, that is $x=A X$. Moreover, it is worth noting that the $l_{0}$ norm can promote the sparsity of the solution to the greatest extent, but since the sparsity regularized least squares cost function based on the $l_{0}$ norm is proved to be a Non-deterministic Polynomial (NP)-hard problem, it is difficult to obtain the optimal solution. Therefore, the $l_{1}$ norm instead of the $l_{0}$ norm is widely used as a regularizer, since it can induce sparsity effectively while the optimal solution can be obtained easily. However, $l_{1}$ norm tends to underestimate non-zero amplitude of the signal, which causes the underestimation of the signal of interest. Consequently, to further induce sparsity and to get over the shortcomings of the $l_{1}$ norm which tends to underestimate non-zero amplitude of the signal [37], various NPFs $\varphi$ are utilized. The detailed instructions about NPFs can be found in [37]. In this section, the $l_{1}$ norm and several typical NPFs that can easily ensure the convexity of the regularized least squares cost function and effectively promote the sparsity of the solution are plotted in Figure 3. In the NPFs shown in Figure 3, 'atan' is more similar to the $l_{0}$ norm than the other NPFs, which means the 'atan' function induces the sparsity more strongly than others [23]. Furthermore, it can effectively maintain the amplitude of the signal of interest. Therefore, to induce the sparsity of STFT coefficient matrix, the 'atan' penalty function is utilized in this paper.

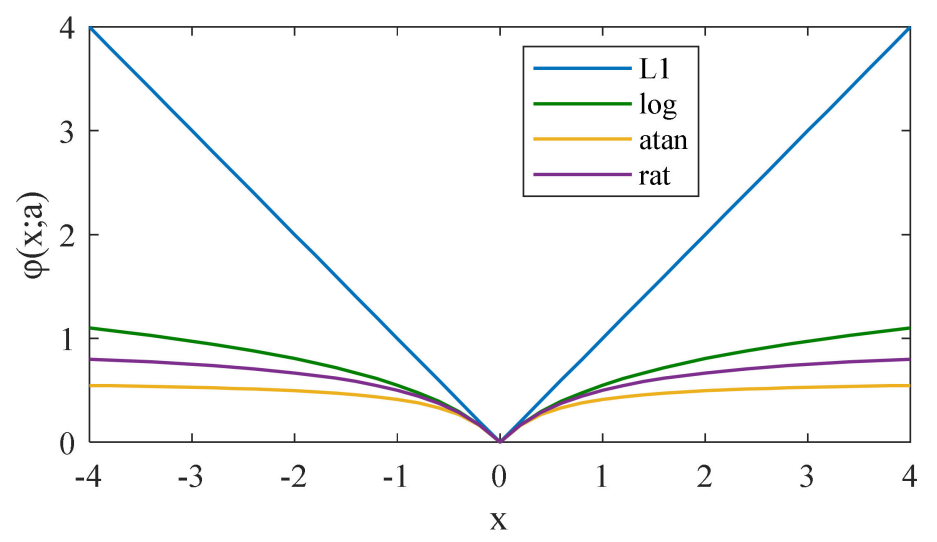

Figure 3. Penalty function $\varphi$.

\subsection{Convexity Condition}

As mentioned above, to maintain the amplitude of a denoised signal, and to further induce sparsity of the solution of optimization problem (2), the penalty function 'atan' is utilized. Therefore, in order to obtain the global optimal solution, the non-convex penalty parameter $a$ is studied to ensure the convexity of optimization function $F(\mathrm{x})$. In the optimization function $F(\mathrm{x}), \sum_{i=1}^{k} \sigma_{i}(X)$ is essentially $l_{1}$ norm of the SVs of the matrix $X$, so it is only necessary to ensure that the remaining part of the optimization problem (2) is convex to guarantee the convexity of whole function, since the convexity 
of the sum of two convex functions does not change. According to Lemma 2 in [38], the convexity of optimization function $F(\mathrm{x})$ can be ensured if:

$$
0<a \leq \frac{1}{\lambda_{1}}
$$

\subsection{Algorithm Derivation}

In this section, variable splitting and the ADMM are used to solve the proposed optimization problem. After variable splitting, objective function (2) can be rewritten as:

$$
\begin{aligned}
& \left\{X^{*}, Z^{*}\right\}=\arg \min _{X \in R^{m \times n}}\left\{F(X):=\|Y-X\|_{F}^{2}+\lambda_{0} \sum_{i=1}^{k} \sigma_{i}(Z)+\lambda_{1} \sum_{m_{1}=1}^{M_{1}} \sum_{m_{2}=1}^{M_{2}} \phi\left(X_{m_{1}, m_{2}} ; a\right)\right\} \\
& \text { s.t. } X=Z .
\end{aligned}
$$

Then, the constrained optimization problem (4) can be converted to the following simple iterative procedures by using the ADMM algorithm, where the scalar augmented Lagrangian parameter is represented by $\mu$. When $\mu>1$, the convergence of the ADMM can be guaranteed [38].

$$
\begin{gathered}
X \leftarrow \underset{X}{\operatorname{argmin}} \frac{1}{2}\|Y-X\|_{F}^{2}+\frac{\mu}{2}\|X-(Z+D)\|_{F}^{2}+\lambda_{1} \sum_{m_{1}=1}^{M_{1}} \sum_{m_{2}=1}^{M_{2}} \phi_{\varepsilon}\left(X_{m_{1}, m_{2}} ; a\right) \\
Z \leftarrow \underset{Z}{\operatorname{argmin}} \frac{\mu}{2}\|X-(Z+D)\|_{F}^{2}+\lambda_{0} \sum_{i=1}^{k} \sigma_{i}(Z) \\
D \leftarrow D-(X-Z)
\end{gathered}
$$

For the sub-problem (5), if constant terms are ignored it can be rewritten as:

$$
X \leftarrow \underset{X}{\operatorname{argmin}}\left\{\frac{1}{2}\left\|\frac{1}{1+\mu}(Y+\mu(Z+D))-X\right\|_{F}^{2}+\frac{\lambda_{1}}{1+\mu} \sum_{m_{1}=1}^{M_{1}} \sum_{m_{2}=1}^{M_{2}} \phi_{\varepsilon}\left(X_{m_{1}, m_{2}} ; a\right)\right\}
$$

The above equation is strictly convex when the convex condition analyzed above is satisfied, and it can be solved by executing the proximal operator associated with $\varphi$, i.e.,

$$
X \leftarrow \operatorname{prox}_{\phi}\left(Y ; \lambda_{1} /(1+\mu), a\right)
$$

The sub-problem (6) as a standard nuclear norm minimization (NNM) problem, and its global optimal solution can be obtained by using the "singular value thresholding" (SVT):

$$
Z \leftarrow U \bullet \operatorname{soft}\left(\Sigma, \lambda_{0}\right) \bullet V^{T}
$$

where, $Y=U \Sigma V^{T}$ is the SVD of $Y$; and soft(...) is the soft-thresholding function [39].

$$
\operatorname{soft}(x, T)=\operatorname{sign}(x) \bullet \max (|x|-T, 0)
$$

By iteratively solving (9), (10) and (7), the proposed objective function (2) can be finally solved.

\subsection{Correlated Kurtosis-Based Thresholding (CKT) Scheme Proposed in This Paper}

As mentioned above, the problem in (6) can be solved easily by applying the SVT. But as stated in Equation (11), SVT preserves the SVs greater than $\lambda_{0}$ and the other SVs are set to zero [37]. This means that the SVT preserves components with large SVs in the matrix. However, it is worth noting that components with large SVs are not necessarily rich in information, especially when the repetitive 
impulses are weak. Therefore, when the bearing's fault features are weak or there are other large interferences such as HCs in the signal, SVT tend to ignore the fault features. Based on the above analysis, in the proposed FISLRA, the CKT scheme is used to instead SVT to save the components with a large amount of fault information. In the CKT method, the correlation kurtosis (CK) which is extensively applied to the fault diagnosis is used as an indicator for measuring the amount of fault information.

$$
\mathrm{CK}_{M}(T)=\frac{\sum_{n=1}^{N}\left(\Pi_{m=0}^{M} y_{n-m T}\right)^{2}}{\left(\sum_{n=1}^{N} y_{n}^{2}\right)^{M+1}}
$$

where $y$ is the signal with length $N$. In this paper, shift number $M=2$ is represented and period of interested is denoted by $T$ which can be easily calculated according to fault characteristic frequency (FCF). A detailed description of CK can be found in [40]. CK measures both the kurtosis and periodicity of the signal, thus it is more conducive for the extraction of repetitive impulses from noisy signal than indicators that only emphasize impact or periodicity.

$$
\mathrm{Z} \leftarrow U \bullet C K T(\Sigma, \mathrm{CK}, k) \bullet V^{T}
$$

where $C K T(\Sigma, C K, k)$ indicates that the SVs with a large amount of fault information are retained, and others are discarded; $k$ is the number of the components to be saved. According to simulation analysis and experimental verification, it is recommended to set it to $10 \%-50 \%$ of the number of SVs. In the experimental verification and simulation analysis section of this article, $k$ is set it to $20 \%$ of the number of SVs. In addition, based on the analysis above, the scalar-augmented Lagrangian parameter $\mu=1.1$ is used in this paper. Non-convex penalty parameter $a$ is set to $1 / \lambda_{1}$ to maximize the sparsity of the solution while ensuring convexity. Furthermore, the regularization parameter $\lambda_{1}$ which has a great influence on the processing result of FISLRA must be appropriately selected according to the signal itself. As mentioned above, the purpose of this article is to extract the fault information caused by bearing defects, and CK is an excellent criterion to indicate the amount of fault information, then the parameter $\lambda_{1}$ that corresponds to the maximum CK of the denoised signal is regarded as the optimal value in this article.

The algorithm of the proposed FISLRA for extracting fault features is summarized in Algorithm 1.

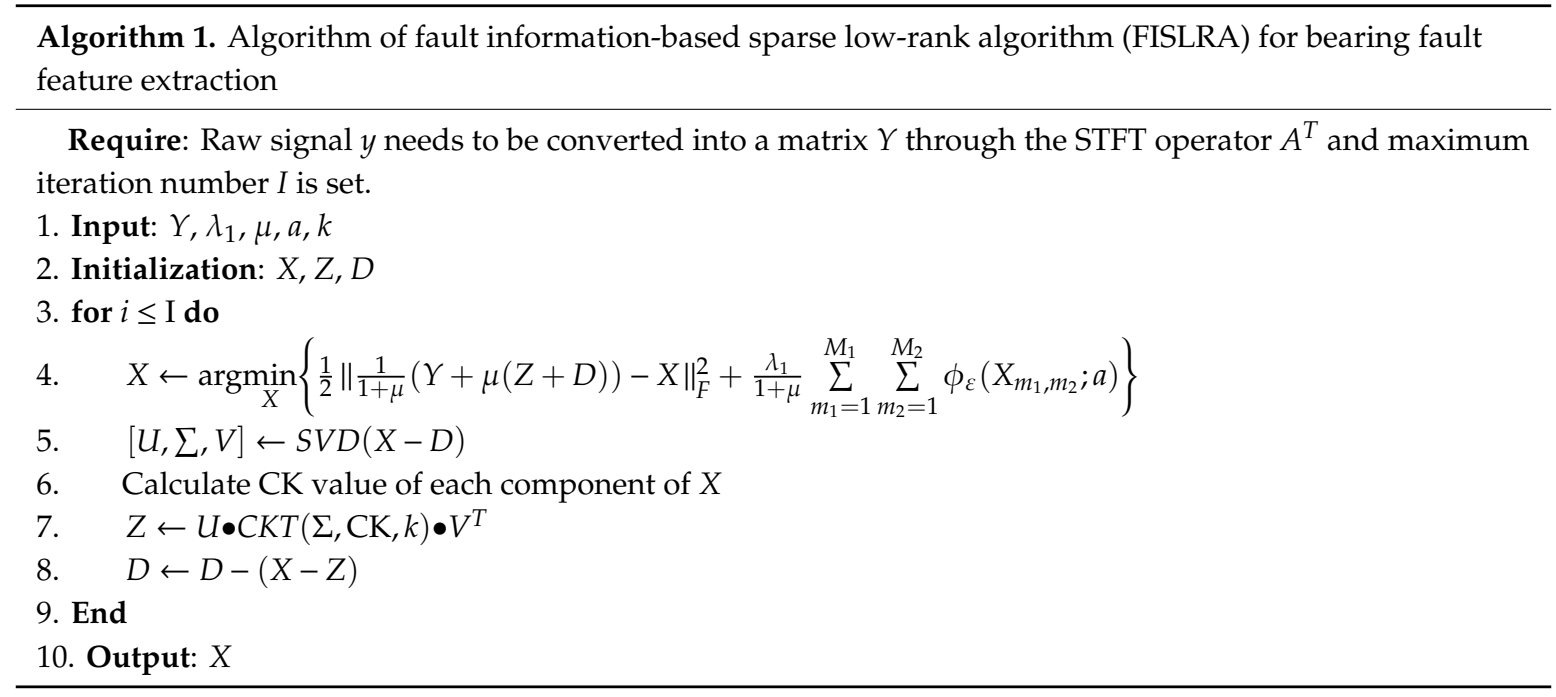

\section{Simulation Analysis}

In the simulation part, two simulated signals with outer race defect are employed to test the utility of the proposed FISLRA. To further demonstrate the advantage of FISLRA, comparative analysis 
of FISLRA and two other methods (TSLRM and SK) is performed. As mentioned above, TSLRM is susceptible to HCs. Therefore, two simulated signals $y_{1}$ and $y_{2}$ are analyzed to test the superiority of FISLRA relative to TSLRM. The results obtained are compared. The vibration signal $y_{1}$ contains Harmonic Components $h(\mathrm{t})$, Repetitive Transients $d(\mathrm{t})$, Random Shocks $r(\mathrm{t})$ and white noise $n(\mathrm{t})$. The vibration signal $y_{2}$ contains the fault-induced impulse $d(\mathrm{t})$, random shocks $r(\mathrm{t})$, white noise $n(\mathrm{t})$ but no HCs. The simulated signal $y_{1}$ is modeled as follows:

$$
\begin{aligned}
y_{1}(t) & =d(t)+h(t)+r(t)+n(t) \\
= & \sum_{i} D_{i} S_{d}\left(t-i T_{d}-\tau_{i}\right)+\sum_{n} A_{n} \cos \left(2 \pi f_{n} t+\alpha_{n}\right) \\
+ & \sum_{i} R_{i} S_{i}\left(t-T_{i}\right)+n(t) \\
& S(t)=e^{-\alpha_{r} t} \sin \left(2 \pi f_{r} t\right)
\end{aligned}
$$

where the first part denotes the repetitive transients $d(\mathrm{t}) . D_{i}$ represents the amplitude of the $i$ th impulse; $T_{d}$ is the fault period of the repetitive transients; $\tau_{i}$ denotes the random slip, and usually $\tau_{i}$ is set to $1 \%-2 \%$ of the fault period; $\alpha_{r}$ is damping ratio of the impulse response and $f_{r}$ is the resonance frequency. The second part denotes the harmonic components $h(\mathrm{t})$ generated by gearbox or rotor. $\alpha_{n}$ is the initial phase and $A_{n}$ is the amplitude; $f_{n}$ stands for the rotating frequency. The third item represents the random shocks $r(\mathrm{t})$, where $T_{i}$ and $R_{i}$ are occurrence time and amplitude of the $i$ th shock, respectively. The white noise is represented by $n(t)$. The corresponding sub-components of simulated signal $y_{1}$ are drawn in Figure $4 a-d$. Time length of simulated data is $1 \mathrm{~s}$ and the sampling frequency is $20,000 \mathrm{~Hz}$. Table 1 lists the settings of other parameters.

(a)
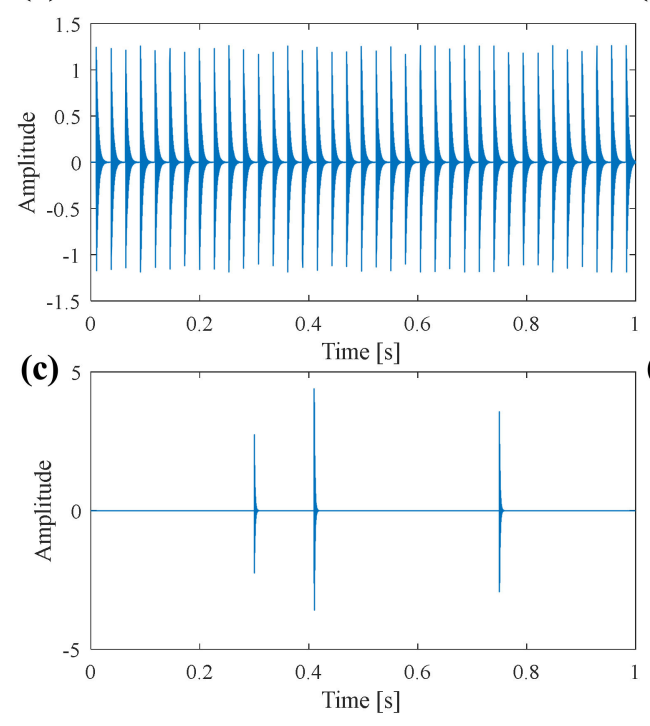

(b)

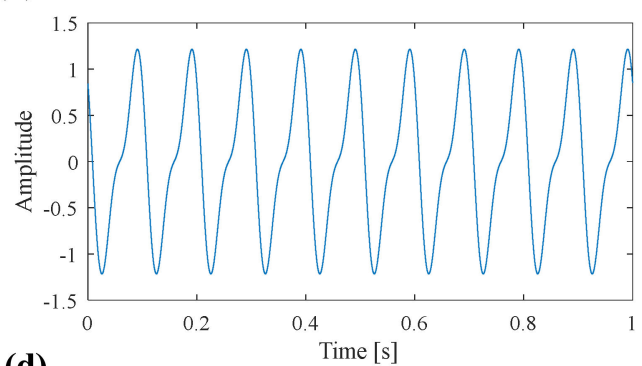

(d)

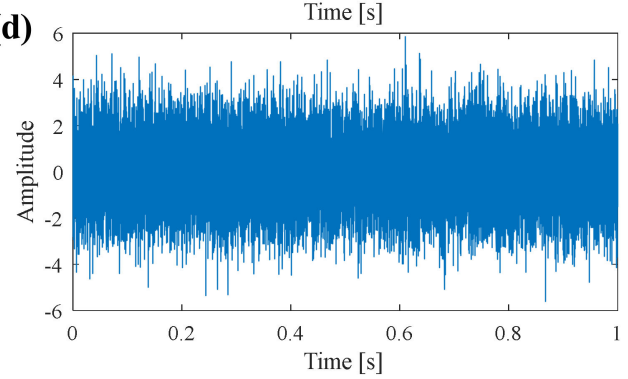

Figure 4. Sub-component of simulated signal $y_{1}$. (a) Repetitive transients $d(\mathbf{t})$; (b) rotor vibration $h(\mathrm{t})$; (c) random shocks $r(\mathrm{t})$; (d) white noise $n(\mathrm{t})$.

Table 1. Parameter setting of signals in simulation analysis.

\begin{tabular}{cccccccccccc}
\hline \multicolumn{3}{c}{ Repetitive Transients } & \multicolumn{4}{c}{ Harmonic Components } & \multicolumn{3}{c}{ Random Shocks } \\
\hline$D_{1}$ & $T_{d}$ & $f_{r}$ & $\alpha_{r}$ & $f_{1}$ & $f_{2}$ & $A_{1}$ & $A_{2}$ & $\alpha_{1}$ & $\alpha_{2}$ & $f_{r}$ & $\alpha_{r}$ \\
1.3 & $1 / 37$ & 2500 & 300 & 10 & 20 & 1 & 0.4 & $\pi / 3$ & $\pi / 6$ & 6000 & 800 \\
\hline
\end{tabular}

The composition and parameter settings of the vibration signal $y_{2}$ are exactly the same as those of the vibration signal $y_{1}$, except that HCs are not included. After adding the white noise with SNR of $-3 \mathrm{~dB}$, the waveform of the simulated signal $y_{2}$ is demonstrated in Figure 5 , of which fault 
features are disturbed by noise. Thus, TSLRM and FISLRA are used to extract fault features from the simulated signal $y_{2}$. The results obtained by applying TSLRM and FISLRA are demonstrated in Figure 6 . The repetitive transients are successfully extracted by both methods, so the ability of the method based on sparse low rank model for fault detection can be proved. The difference is that the extracted fault feature components obtained by TSLRM are disturbed by random shocks (shown in Figure 4c) that are marked with red dots, but the results obtained by the proposed FISLRA are not disturbed by random shocks. This shows that the robustness of the proposed method FISLRA is better against random impact interference than TSLRM.

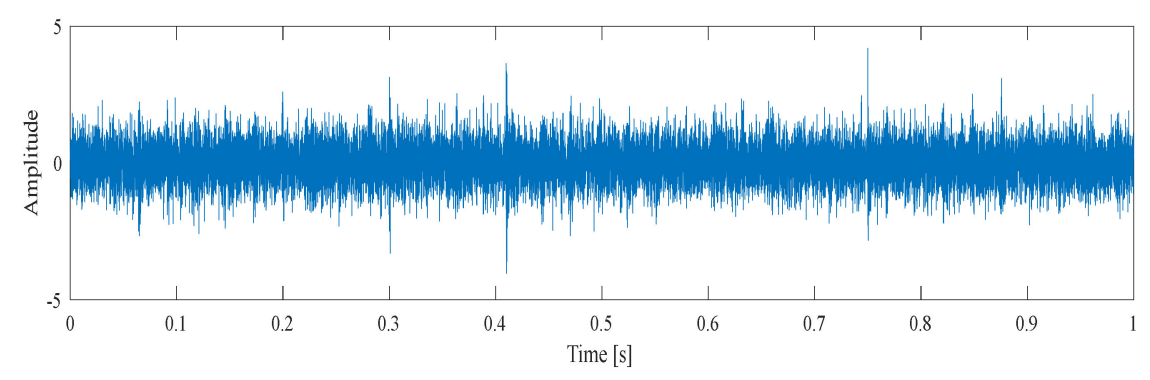

Figure 5. Simulated signal $y_{2}$.

(a)

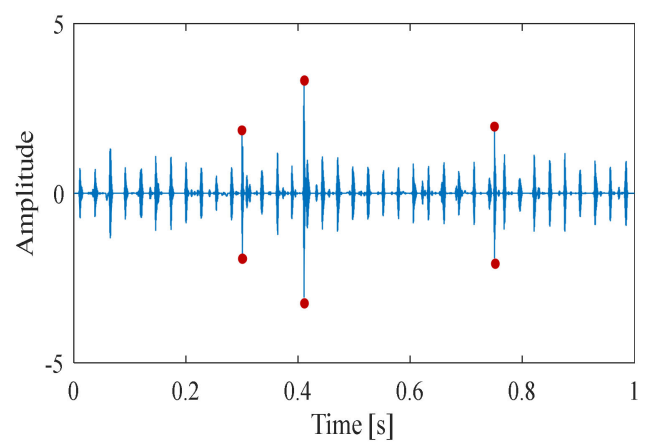

(b)

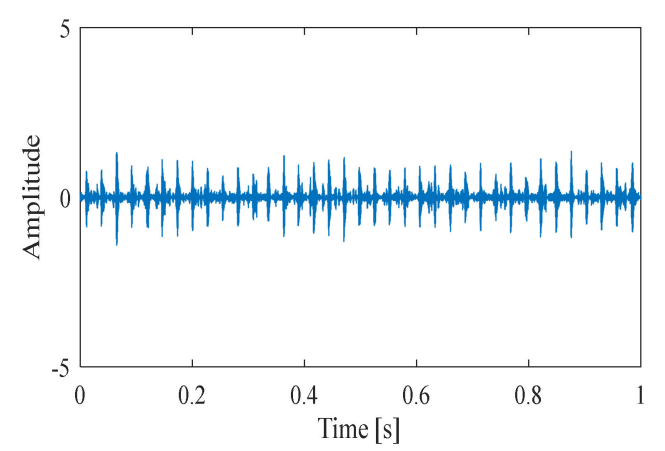

Figure 6. Simulated signal $y_{2}$. (a) Analysis result of the traditional sparse low-rank model (TSLRM);

(b) analysis result of the fault information-based sparse low-rank algorithm (FISLRA).

To further certify the superiority of FISLRA over TSLRM, the simulated signal $y_{1}$ is analyzed. The simulated signal $y_{1}$ and the relevant envelope spectrum are displayed in Figure 7 . In the waveform, the defect impulses are overwhelmed by heavy noises. In envelope spectrum, the frequencies corresponding to the HCs (marked by red circle) are apparent, but the FCF is overwhelmed by other frequency lines. Therefore, it is difficult to judgment whether the bearing has failed from the raw signal.

(a)

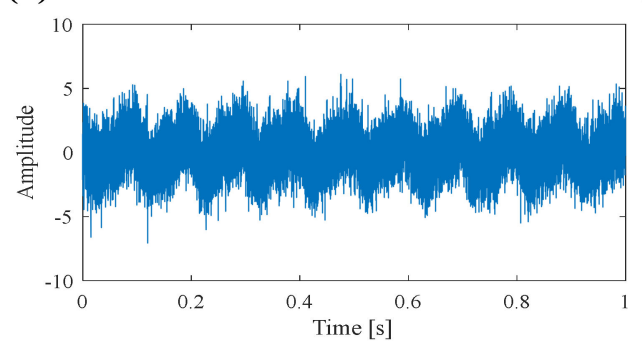

(b)

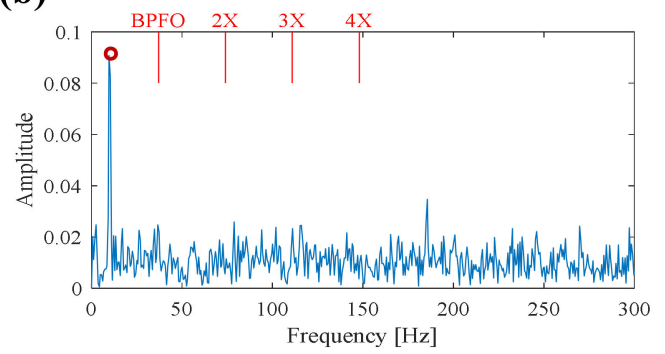

Figure 7. Simulated signal $y_{1}$. (a) Amplitude spectrum; (b) envelope spectrum.

Next, the simulated signal $y_{1}$ is processed by FISLRA and TSLRM. Figure 8 represents the results obtained using the FISLRA, in which the extracted repetitive transients are distinct. In the envelope 
spectrum, FCF and its harmonics can be recognized clearly. Therefore, there is no doubt that the FISLRA can effectively abstract the repetitive transients from the noisy signal even in the case of interference from HCs. Moreover, to explore the computational efficiency of the proposed FISLRA, a time cost analysis is conducted on an Intel Core i7-9750H desktop computer with a $4.5 \mathrm{GHz} \mathrm{CPU}$. For the simulated signal with a length of 20,000 data points, the average time cost of the proposed method FISLRA is $4 \mathrm{~s}$.

(a)

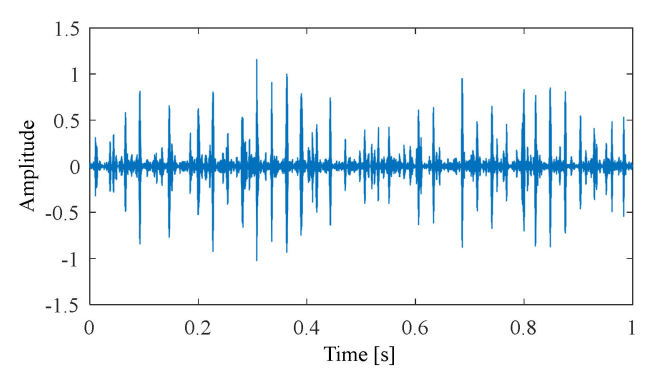

(b)

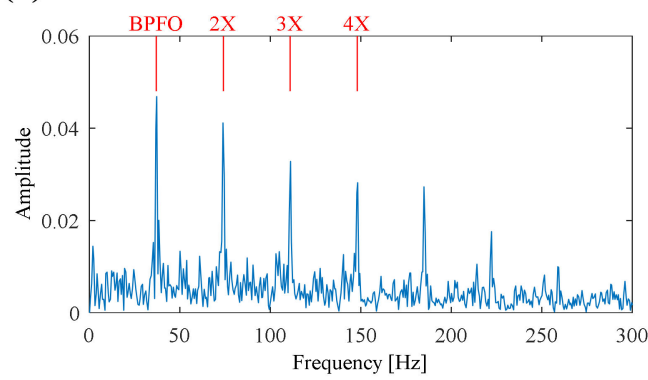

Figure 8. Fault features extracted by FISLRA. (a) Amplitude spectrum; (b) envelope spectrum.

Then, the same simulated signal $y_{1}$ is analyzed by using the TSLRM. Figure 9a presents the resultant denoised signal, where the HCs is obvious but no obvious defect impulses can be observed from the amplitude spectrum. And as indicated in Figure $9 \mathrm{~b}$, the envelope spectrum also fails to provide useful information for fault diagnosis. Moreover, rotating frequency and its harmonics ( $1 X$ and $2 X$ ) are very dominant in envelope spectrum, which means that TSLRM is interfered with by the HCs during the extraction of fault features. Therefore, there is no doubt that the FISLRA can effectively abstract the repetitive transients from the noisy signal even in the case of interference from HCs. The superiority of FISLRA over TSLRM can be certified in the section of simulation analysis.

(a)

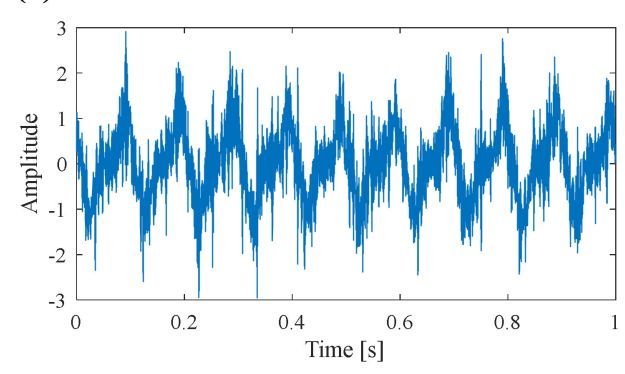

(b)

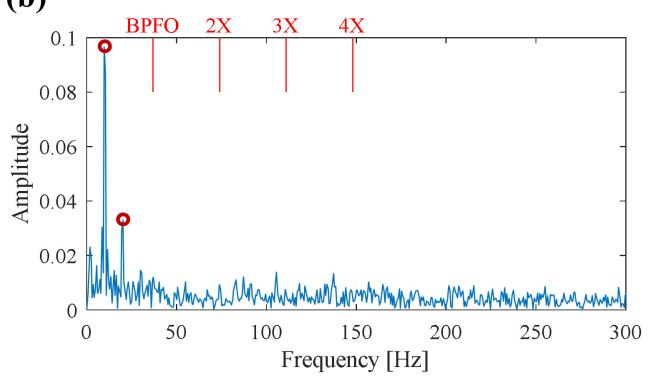

Figure 9. Fault features extracted by the TSLRM. (a) Amplitude spectrum; (b) envelope spectrum.

Furthermore, to further highlight the effectiveness of the FISLRA, the SK as a widely used and state-of-art method is applied to deal with the same simulated signal $y_{1}$. Figure 10a displays the kurtogram of the simulated signal, the optimal frequency band with a bandwidth of $312.5 \mathrm{~Hz}$ and the center frequency of $6093.75 \mathrm{~Hz}$ flagged by a red rectangle is located in the kurtogram. The relevant filtered signal is indicated in Figure 10b, from which fault information cannot be found intuitively, and ball pass frequency of outer race (BPFO) and its harmonics are overwhelmed by interference frequencies in envelope spectrum. Therefore, SK fails to extract fault information. The failure result from the assumption that the fault impulses occur in the frequency band with the highest kurtosis. Research has found that kurtosis is more sensitive to a single impulse than to periodic impulses. Therefore, the SK method is susceptible to interference from non-Gaussian signals and random shocks. 
(a)

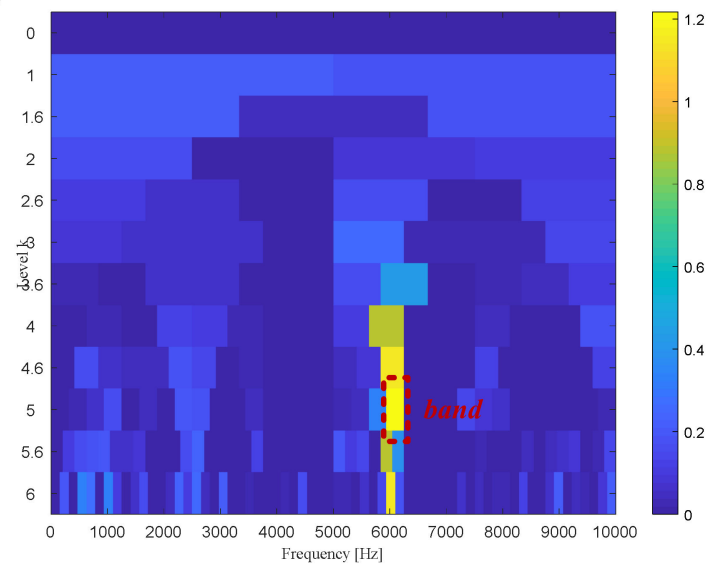

(b)

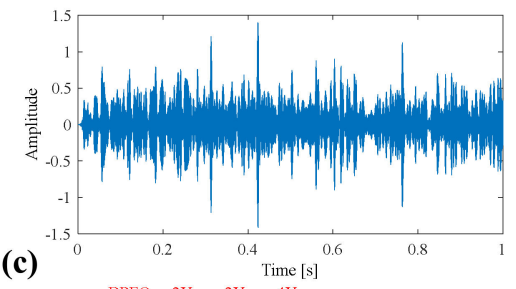

(c)

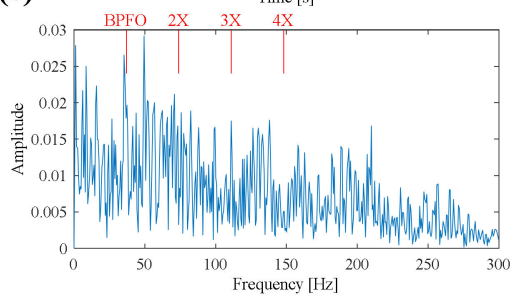

Figure 10. Fault features extracted by SK. (a) Kurtogram of the simulated signal $y_{1} ;$ (b) denoised signal; (c) envelope spectrum.

\section{Experimental Verification}

In this section, two experimental signals collected from a locomotive bearing test rig are used to further verify the validity of the FISLRA method. The test rig mainly consists of a driving wheel, hydraulic motor and locomotive wheel as presented in Figure 11. The outer race of the bearing is driven while the inner race is fixed. Therefore, the envelope spectrum of collected vibration signal is expected to contain sidebands when an outer race defect occurs. During the experiment, the experimental signal is collected at the sampling frequency of $76,800 \mathrm{~Hz}$ and the time length of the data is $1 \mathrm{~s}$. Furthermore, the speed signal can be acquired by a tachometer while collecting the vibration signal. Parameters of tested bearing in experimental verification are listed in Table 2 . In case 1 , the outer race defective REB as shown in Figure 12a is mounted on the test rig. In case 2, the REB with an inner race defect as shown in Figure $12 \mathrm{~b}$ is mounted on the test rig. Therefore, in the measured experimental signals, there should be repetitive transients produced by the defect in the measured test data.

(a)

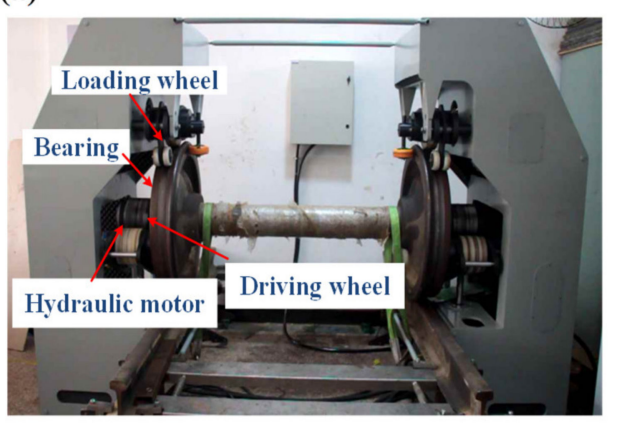

(b)

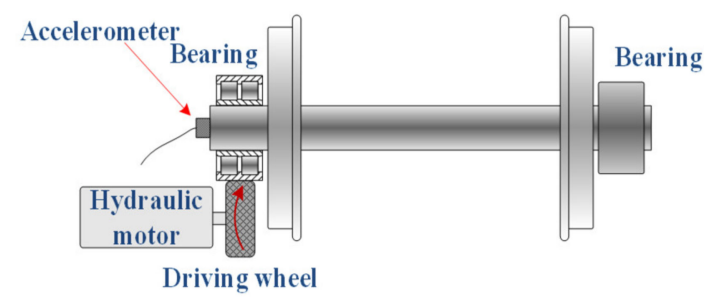

Figure 11. The test rig. (a) actual installation diagram; (b) schematic.

Table 2. Parameters of tested bearing in experimental verification.

\begin{tabular}{cccc}
\hline Number of Rollers & Pitch Diameter $(\mathbf{m m})$ & Roller Diameter (mm) & Contact Angle (Degree) \\
\hline 20 & 180 & 23.775 & 9 \\
\hline
\end{tabular}

\subsection{Case 1: Bearing Outer Race Fault Detection}

In case 1 , the outer ring defective REBs is mounted on the test rig. The acquired vibration signal is shown in Figure 13a. However, due to the interference of the interference component in the signal, the defect-induced impulse cannot be detected obviously from the time waveform. Meanwhile, 
no significant FCF is observed in the envelope spectrum as displayed in Figure 13b. Therefore, in order to acquire more useful fault information for bearing fault diagnosis, further signal processing is needed.

(a)

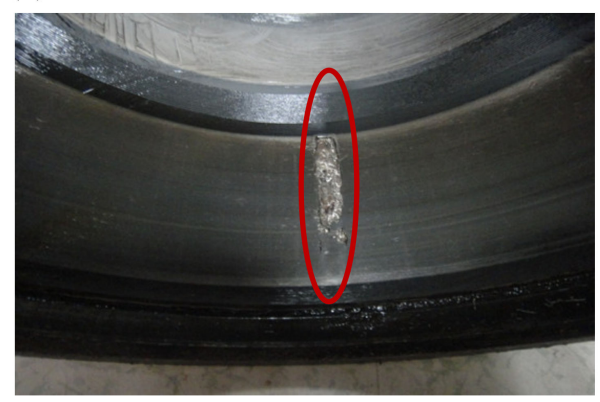

(b)

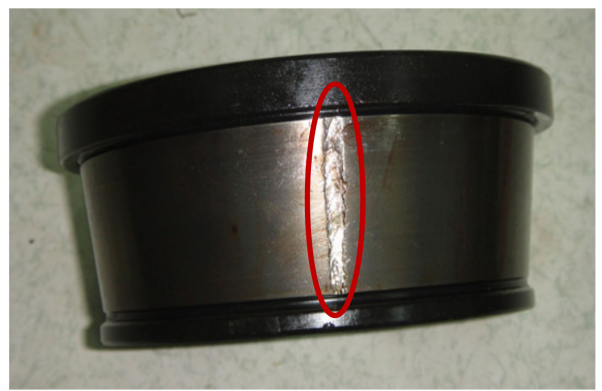

Figure 12. (a) The tested bearing with an outer race fault; (b) the tested bearing with an inner race defect.

(a)

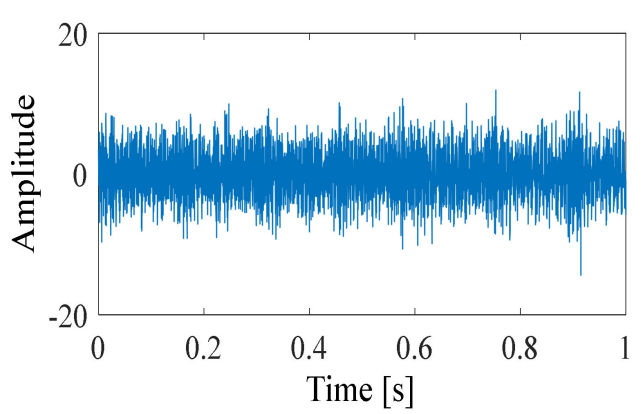

(b)

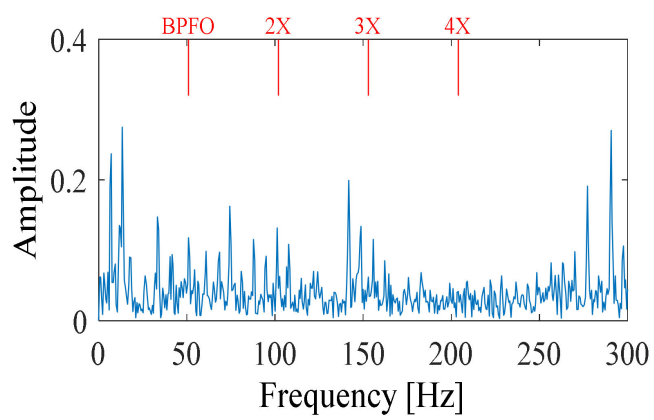

Figure 13. (a) Measured test data; (b) envelope spectrum.

Firstly, the TSLRM method is applied for the extraction of the weak fault features from the measured test data with outer race defect, and the fault features extracted by TSLRM are illustrated in Figure 14. However, the fault-related information is difficult to observe from the waveform. Furthermore, the BPFO and its multipliers are disturbed by other frequencies. Therefore, the denoised signal obtained by the TSLRM method is unsatisfactory. The results obtained do not provide useful information on bearing failure and, therefore, may lead to missed and misdiagnosed bearing failures.

(a)

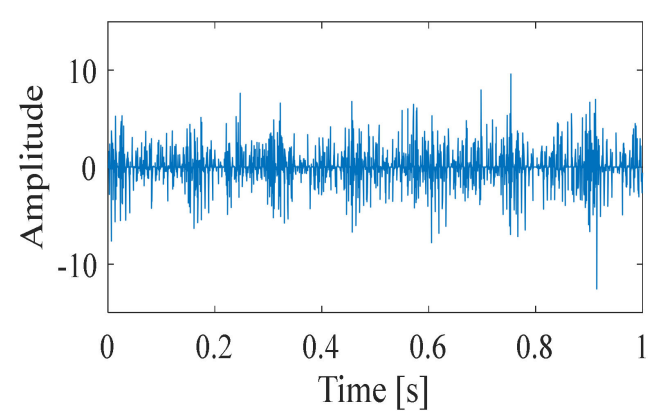

(b)

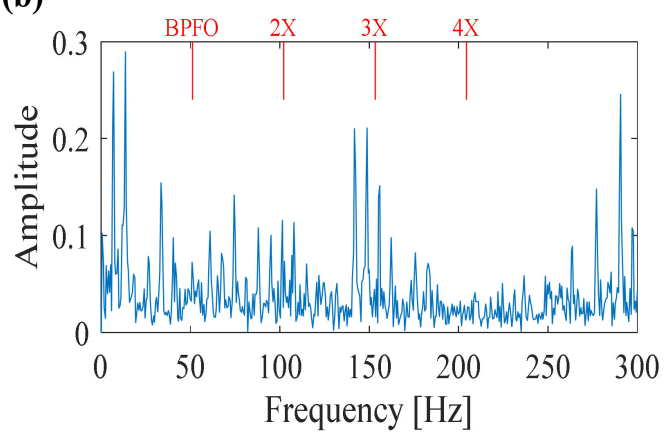

Figure 14. Fault features extracted by TSLRM method. (a) Amplitude spectrum; (b) envelope spectrum.

For comparison, the FISLRA is conducted for the extraction of fault features, and the processed results of the measured test data are demonstrated in Figure 15. In waveform, the repetitive transients are successfully extracted, and the BPFO and its multipliers can be clearly observed in the related envelope spectrum. Therefore, the extracted fault features provide strong evidence for the occurrence of the outer ring fault. The above case analysis shows that TSLRM did not successfully extract fault features, while FISLRA successfully extracted fault features. 
(a)

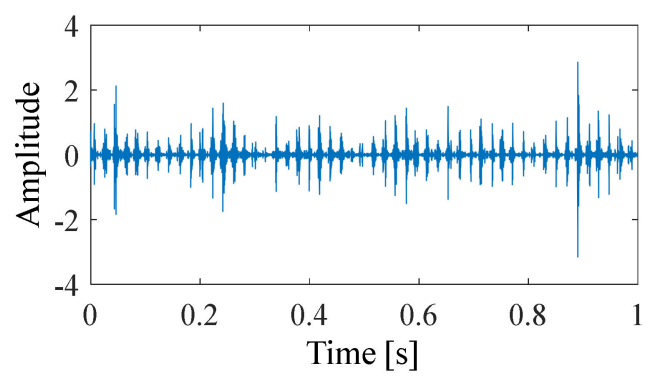

(b)

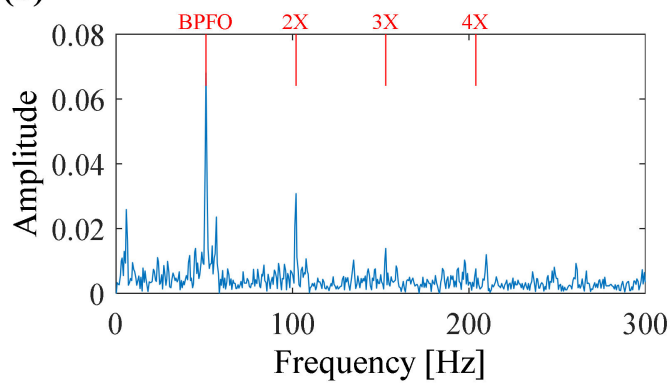

Figure 15. Fault features extracted by the FISLRA. (a) Amplitude spectrum; (b) envelope spectrum.

\subsection{Case 2: Bearing Inner Race Fault Detection}

In case 2, the REBs with an outer race defect were mounted on the test rig. The measured test data with inner race defect are used to further investigate the superiority of FISLRA in detecting the bearing fault. The measured test data and their envelope spectrum are illustrated in Figure 16. But the amplitude spectrum and the envelope spectrum do not offer any powerful fault information.

(a)

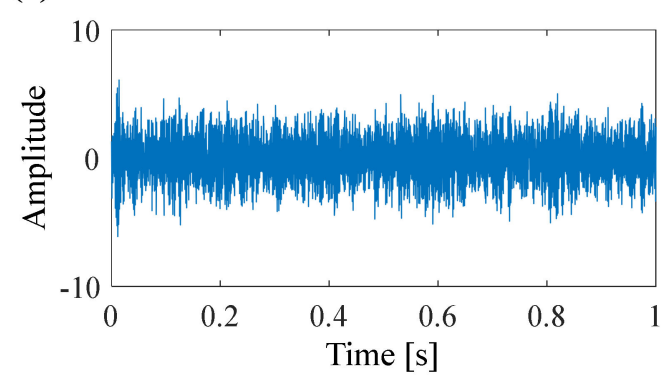

(b)

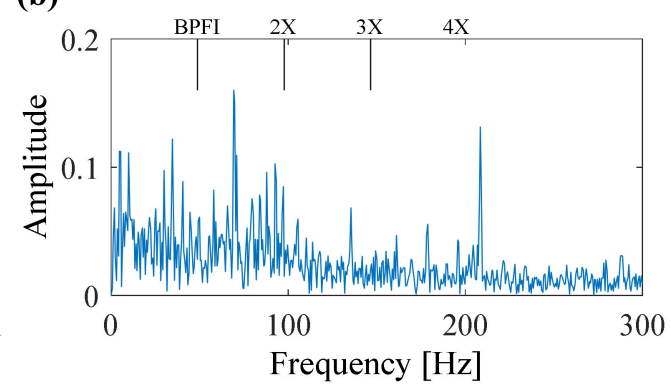

Figure 16. (a) Measured test data; (b) envelope spectrum.

Then, FISLRA is performed on the raw signal to provide basic fault information for bearing condition monitoring and the results are shown in Figure 17. Repetitive transients are successfully extracted, which verify the utility of FISLRA in recovering fault features. Moreover, the envelope analysis is performed for the extracted fault features, and FCF and its multiplier are very distinct in the envelope spectrum, which indicates the inner race of the rolling bearing is faulty. For comparative analysis, the results obtained by TSLRM are displayed in Figure 18. Similar to case 1, the results of fault feature extraction obtained by TSLRM are unsatisfactory. The above analysis based on experimental data shows that FISLRA is an effective tool for condition monitoring of the bearing. In addition, comparative analysis also proves the superiority of the FISLRA over TSLRM in the extraction of fault features.

(a)

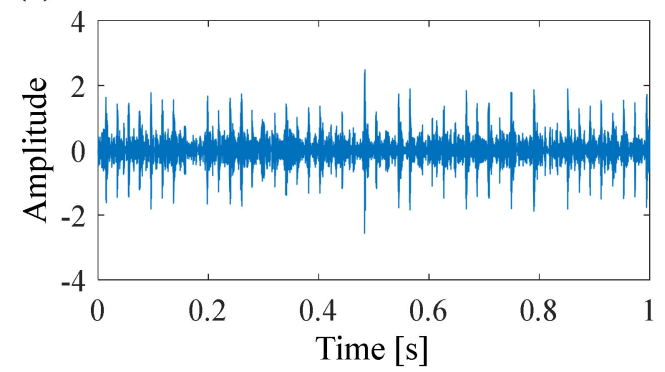

(b)

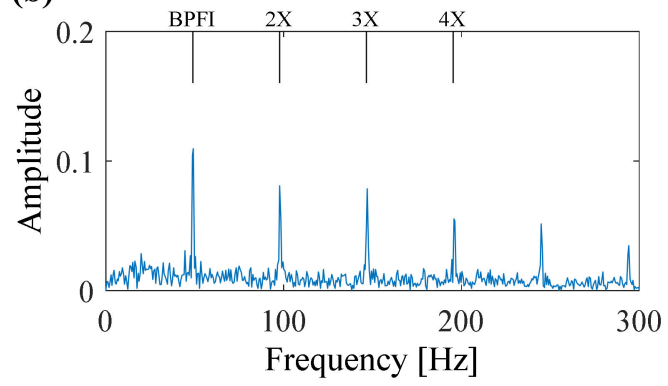

Figure 17. Fault features extracted by FISLRA. (a) Amplitude spectrum; (b) envelope spectrum. 
(a)

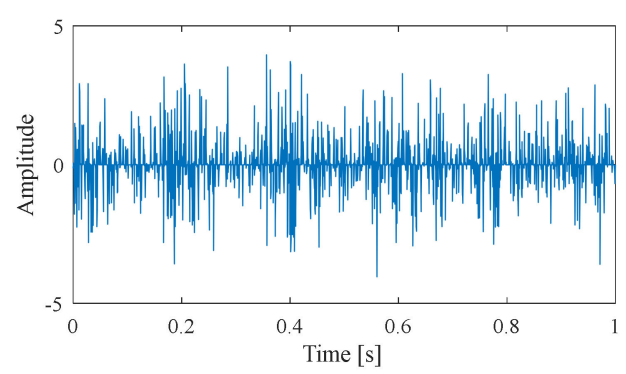

(b)

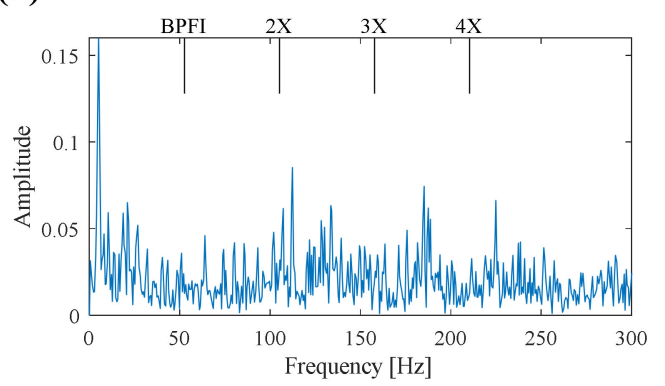

Figure 18. Fault features extracted by TSLRM. (a) Amplitude spectrum; (b) envelope spectrum.

\section{Conclusions}

According to the low-rank characteristic and sparsity of fault features in the time-frequency domain, this paper proposes a novel method FISLRA based on a low-rank sparse matrix estimation for the diagnosis of REBs. Concretely, a sparse and low-rank model is formulated in the time-frequency domain. Then, a fast-converging algorithm is derived based on ADMM to solve the formulated model. Moreover, to further highlight the periodical transients, a CKT scheme proposed in this paper is also incorporated to solve the proposed low-rank spare model. With the proposed method, the weak fault features indicating the occurrence of the bearing are successfully extracted. Moreover, the effectiveness and advantages of the FISLRA are validated in detecting the bearing fault by comparing it with TSLRM and SK. In short, FISLRA can be seen as an enhanced method of the TSLRM method. Compared with the TSLRM method which is susceptible to HCs, FISLRA can effectively abstract the fault features from a noisy signal even if disturbed by HCs. However, the setting of the parameters in the FISLRA method is worthy of deeper research. In the field of bearing fault diagnosis, low-rank sparse matrix estimation is currently in the early stages of research, and the setting of parameters is still an open topic that requires further research. Therefore, we will conduct research on parameter settings in future research work. Moreover, we have found through preliminary research that the gearbox fault features also exhibit a low-rank property and sparsity in the time-frequency domain. Therefore, we will conduct further research on the gearbox fault feature based on low-rank sparse matrix estimation in the future.

Author Contributions: B.W. proposed the method FISLRA. Y.L. and B.W. analyzed the signal and wrote the draft manuscript, X.Z. and R.D. provided some valuable advice. All authors have read and agreed to the published version of this manuscript.

Funding: This research was funded by the National Natural Science Foundation of China grant number 51575424, the National Key Research and Development Program of China grant number 2019YFB1311903 and the Joint Foundation of the Ministry of Education grant number 6141A02022113. And the APC was funded by the National Natural Science Foundation of China grant number 51575424.

Conflicts of Interest: The authors declare no conflicts of interest.

\section{References}

1. Guzmán-Rabasa, J.A.; López-Estrada, F.-R.; González-Contreras, B.M.; Valencia-Palomo, G.; Chadli, M.; Pérez-Patricio, M. Actuator fault detection and isolation on a quadrotor unmanned aerial vehicle modeled as a linear parameter-varying system. Meas. Control 2019, 52, 1228-1239. [CrossRef]

2. Arpaia, P.; Cesaro, U.; Chadli, M.; Coppier, H.; De Vito, L.; Esposito, A.; Gargiulo, F.; Pezzetti, M. Fault detection on fluid machinery using Hidden Markov Models. Measurement 2020, 151, 107126. [CrossRef]

3. Akhenak, A.; Chadli, M.; Maquin, D.; Ragot, J. Sliding mode multiple observer for fault detection and isolation. In Proceedings of the 42nd IEEE International Conference on Decision and Control (IEEE Cat. No. 03CH37475), Maui, HI, USA, 9-12 December 2003; IEEE: New York, NY, USA, 2004; Volume 1.

4. Akhenak, A.; Chadli, M.; Maquin, D.; Ragot, J. State estimation via multiple observer with unknown inputs: Application to the three tank system. In Proceedings of the 5th IFAC Symposium on Fault Detection, Supervision and Safety for Technical Processes, Safeprocess 2003, Washington, DC, USA, 9-11 June 2003. 
5. El Hajjaji, A.; Chadli, M.; Oudghiri, M.; Pages, O. Observer-based robust fuzzy control for vehicle lateral dynamics. In Proceedings of the 2006 American Control Conference, Minneapolis, MN, USA, 14-16 June 2006; IEEE: New York, NY, USA, 2006.

6. Qiao, W.; Lu, D. A Survey on Wind Turbine Condition Monitoring and Fault Diagnosis_Part II: Signals and Signal Processing Methods. IEEE Trans. Ind. Electron. 2015, 62, 6546-6557. [CrossRef]

7. Cai, G.; Selesnick, I.W.; Wang, S.; Dai, W.; Zhu, Z. Sparsity-enhanced signal decomposition via generalized minimax-concave penalty for gearbox fault diagnosis. J. Sound Vib. 2018, 432, 213-234. [CrossRef]

8. Cai, B.; Zhao, Y.; Liu, H.; Xie, M. A Data-Driven Fault Diagnosis Methodology in Three-Phase Inverters for PMSM Drive Systems. IEEE Trans. Power Electron. 2017, 32, 5590-5600. [CrossRef]

9. Shao, H.; Jiang, H.; Zhang, H.; Duan, W.; Liang, T.; Wu, S. Rolling bearing fault feature learning using improved convolutional deep belief network with compressed sensing. Mech. Syst. Signal Process. 2018, 100, 743-765. [CrossRef]

10. Randall, R.B.; Antoni, J. Rolling element bearing diagnostics-A tutorial. Mech. Syst. Signal Process. 2011, 25, 485-520. [CrossRef]

11. Randall, R.B. Vibration-Based Condition Monitoring: Industrial, Aerospace and Automotive Applications; John Wiley \& Sons: Hoboken, NJ, USA, 2011.

12. Antoni, J. The infogram: Entropic evidence of the signature of repetitive transients. Mech. Syst. Signal Process. 2016, 74, 73-94. [CrossRef]

13. Ding, C.; Zhao, M.; Lin, J.; Jiao, J. Multi-objective iterative optimization algorithm based optimal wavelet filter selection for multi-fault diagnosis of rolling element bearings. ISA Trans. 2019, 88, 199-215. [CrossRef]

14. Hemmati, F.; Orfali, W.; Gadala, M.S. Roller bearing acoustic signature extraction by wavelet packet transform, applications in fault detection and size estimation. Appl. Acoust. 2016, 104, 101-118. [CrossRef]

15. Antoni, J. The spectral kurtosis: A useful tool for characterising non-stationary signals. Mech. Syst. Signal Process. 2006, 20, 282-307. [CrossRef]

16. Antoni, J. Fast computation of the kurtogram for the detection of transient faults. Mech. Syst. Signal Process. 2007, 21, 108-124. [CrossRef]

17. Dragomiretskiy, K.; Zosso, D. Variational Mode Decomposition. IEEE Trans. Signal Process. 2013, 62, 531-544. [CrossRef]

18. Lei, Y.; Lin, J.; He, Z.; Zuo, M.J.; Zuo, M.J. A review on empirical mode decomposition in fault diagnosis of rotating machinery. Mech. Syst. Signal Process. 2013, 35, 108-126. [CrossRef]

19. Huang, N.E.; Shen, Z.; Long, S.R.; Wu, M.C.; Shih, H.H.; Zheng, Q.; Yen, N.-C.; Tung, C.C.; Liu, H.H. The empirical mode decomposition and the Hilbert spectrum for nonlinear and non-stationary time series analysis. Proc. R. Soc. A Math. Phys. Eng. Sci. 1998, 454, 903-995. [CrossRef]

20. Feng, Z.; Liang, M.; Chu, F. Recent advances in time-frequency analysis methods for machinery fault diagnosis: A review with application examples. Mech. Syst. Signal Process. 2013, 38, 165-205. [CrossRef]

21. Zhao, Z.; Shuming, W.; Qiao, B.; Wang, S.; Chen, X.; Zhibin, Z. Enhanced Sparse Period-Group Lasso for Bearing Fault Diagnosis. IEEE Trans. Ind. Electron. 2018, 66, 2143-2153. [CrossRef]

22. He, W.; Ding, Y.; Zi, Y.; Selesnick, I.W. Sparsity-based algorithm for detecting faults in rotating machines. Mech. Syst. Signal Process. 2016, 72, 46-64. [CrossRef]

23. He, W.; Chen, B.; Zeng, N.; Zi, Y. Sparsity-based signal extraction using dual Q-factors for gearbox fault detection. ISA Trans. 2018, 79, 147-160. [CrossRef]

24. Selesnick, I.; Farshchian, M. Sparse Signal Approximation via Nonseparable Regularization. IEEE Trans. Signal Process. 2017, 65, 2561-2575. [CrossRef]

25. Bruckstein, A.M.; Donoho, D.L.; Elad, M. From Sparse Solutions of Systems of Equations to Sparse Modeling of Signals and Images. SIAM Rev. 2009, 51, 34-81. [CrossRef]

26. Chen, P.-Y.; Selesnick, I. Translation-invariant shrinkage/thresholding of group sparse signals. Signal Process. 2014, 94, 476-489. [CrossRef]

27. Chen, P.-Y.; Selesnick, I. Group-Sparse Signal Denoising: Non-Convex Regularization, Convex Optimization. IEEE Trans. Signal Process. 2014, 62, 3464-3478. [CrossRef]

28. Ding, Y.; He, W.; Chen, B.; Zi, Y.; Selesnick, I. Detection of faults in rotating machinery using periodic time-frequency sparsity. J. Sound Vib. 2016, 382, 357-378. [CrossRef]

29. Cai, G.; Chen, X.; He, Z. Sparsity-enabled signal decomposition using tunable Q-factor wavelet transform for fault feature extraction of gearbox. Mech. Syst. Signal Process. 2013, 41, 34-53. [CrossRef] 
30. Wang, H.; Chen, J.; Dong, G. Feature extraction of rolling bearing's early weak fault based on EEMD and tunable Q-factor wavelet transform. Mech. Syst. Signal Process. 2014, 48, 103-119. [CrossRef]

31. Selesnick, I.W.; Parekh, A.; Bayram, I. Convex 1-D Total Variation Denoising with Non-convex Regularization. IEEE Signal Process. Lett. 2014, 22, 141-144. [CrossRef]

32. Parekh, A.; Selesnick, I.W. Convex Denoising using Non-Convex Tight Frame Regularization. IEEE Signal Process. Lett. 2015, 22, 1786-1790. [CrossRef]

33. Richard, E.; Savalle, P.-A.; Vayatis, N. Estimation of simultaneously sparse and low rank matrices. arXiv 2012; arXiv:1206.6474.

34. Han, L.; Liu, X.-L. Convex Relaxation Algorithm for a Structured Simultaneous Low-Rank and Sparse Recovery Problem. J. Oper. Res. Soc. China 2015, 3, 363-379. [CrossRef]

35. Zhang, T.; Ghanem, B.; Liu, S.; Xu, C.; Ahuja, N. Low-Rank Sparse Coding for Image Classification. In Proceedings of the 2013 IEEE International Conference on Computer Vision, Sydney, NSW, Australia, 1-8 December 2013; pp. 281-288.

36. Xin, G.; Qin, Y.; Jia, L.-M.; Zhang, S.-J.; Antoni, J. Low-rank and sparse model: A new perspective for rolling element bearing diagnosis. In Proceedings of the 2018 International Conference on Intelligent Rail Transportation (ICIRT), Singapore, 12-14 December 2018; IEEE: New York, NY, USA, 2018.

37. Parekh, A.; Selesnick, I.W. Enhanced Low-Rank Matrix Approximation. IEEE Signal Process. Lett. 2016, 23, 493-497. [CrossRef]

38. Parekh, A.; Selesnick, I.W. Improved sparse low-rank matrix estimation. Signal Process. 2017, 139, 62-69. [CrossRef]

39. Donoho, D. De-noising by soft-thresholding. IEEE Trans. Inf. Theory 1995, 41, 613-627. [CrossRef]

40. McDonald, G.L.; Zhao, Q.; Zuo, M.J. Maximum correlated Kurtosis deconvolution and application on gear tooth chip fault detection. Mech. Syst. Signal Process. 2012, 33, 237-255. [CrossRef]

(C) 2020 by the authors. Licensee MDPI, Basel, Switzerland. This article is an open access article distributed under the terms and conditions of the Creative Commons Attribution (CC BY) license (http://creativecommons.org/licenses/by/4.0/). 